\title{
A Mini Review on Some Latest Break Throughs on Molecular Intervention for Human Diseases
}

\author{
Muhammad Irfan* \\ Department of Biochemistry and Biotechnology, University of Gujrat, Pakistan
}

Received: 眥: November 08, 2018; Published: 制: November 16, 2018

*Corresponding author: Muhammad Irfan, Department of Biochemistry and Biotechnology, University of Gujrat, Pakistan

\begin{abstract}
Finding new targets of biomolecule for diagnostics and therapeutic purposes have been key preference for life sciences researchers from past to the present. Following are few important glimpses from October 2018 in which researcher found new mitochondriaal target for treating colorectal cancer and also found new unique biomarkers for ovarian cancer detection. Evolutionary Pressure against MHC Class II Binding Cancer Mutations was also stated. Purpose of this article is to put light on some important break throughs of molecular interventions for human diseases from October 2018.
\end{abstract}

Keywords: Breast Cancer; Ovarian Cancer; MHC-1; Viruses

Abbreviations: PDT: Photodynamic Treatment; RT: Radiotherapy; nMOFs: Nanoscale Metal- Natural Structures; RDT: Radio Dynamic Treatment; PDT: Photodynamic Therapy; PSs: Photosensitizers; ROS: Reactive Oxygen Species

\section{Introduction}

\section{Nanoscale Metal-Organic Frameworks for Mitochondria- Targeted Radiotherapy-Radiodynamic Therapy in Colorectal Cancer}

Particular conveyance of photochemistry to mitochondria of growth cells can upgrade the adequacy of photodynamic treatment (PDT). Despite the fact that cationic Ru-based photo chemicals gather in mitochondria, they require excitation with less entering short-wavelength photons, restricting their application in PDT. X-beam based disease treatment by nanoscale metal- natural structures (nMOFs) by means of upgrading radiotherapy (RT) and increasing radio dynamic treatment (RDT). In this study researchers reported that Hf-DBB-Ru ([DBB-Ru = bis $\left(2,2^{\prime}\right.$ - bipyridine) $\left(5,5^{\prime}\right.$ di(4-benzoato)- 2,2'- bipyridine) ruthenium (II) chloride] as a mitochondria-focused on nMOF for RT-RDT. Developed from Ru-based photochemicals the cationic structure shows solid mitochondria-focusing on property. Upon X-beam illumination, Hf-DBB-Ru proficiently gives hydroxyl radicals from the Hf6 SBUs and singlet oxygen from the DBB-Ruphotochemicals to prompt RTRDT impacts. Mitochondria-focused on RT-RDT depolarizes the mitochondrial film to start death of the growth cells, enhancing noteworthy relapse of colorectal tumors in mouse models. This work builds up a compelling critriea to specifically target mitochondria with cationic nMOFs for upgraded tumor treatment by means of RT-RDT with low measurements of profoundly infiltrating X-beams.

Photodynamic therapy (PDT) has provided a persuading local cancer treatment by eradicating malignant tumors without damaging surrounding normal tissues. In PDT, molecular oxygen is converted into highly cytotoxic reactive oxygen species (ROS), typically singlet oxygen (102), by photosensitizers (PSs) in their excited states. Since 102 has a short lifetime in biological systems $(\sim 40 \mathrm{~ns})$ and limited radius of diffusion from its site of generation $(<30 \mathrm{~nm})$, PDT selectively targets the locale of a PS at the time of light irradiation. As a consequence of the experiment, the remidal effect of PDT is greatly influenced by localization of PSs in specific subcellular organelles. Several subcellular organelles, including mitochondria, lysosomes, and plasma membranes, have been evaluated as potential PDT targets. In particular, mitochondria have been recognized as a main pharmacological target for cancer treatment due to their central role in mediating cell death. Mitochondria undertake critical functions in various biological processes of cells, including energy production as ATP, body metabolism, and redox status check balance. Mitochondrial inability can cause poor energy supply and activate mitochondriamediated cell death pathways. In cancer, mitochondria play key 
roles on tumor cell proliferation, invasion, and metastasis. Thus, generating 102 inside mitochondria can damage them at the early stage of PDT treatment to maximize the anticancer effect. Promptly new procedures are involved to realize anti-cancer PDT treatment with mitochondria-targeted Ru-based PSs [1].

\section{Integrated Extracellular Microrna Designed to Monitor Ovarian Cancer}

Shortage Of effectual screening methods for timely diagnosis or apt detection of ovarian cancer is one of the significant hindrances in evolving/ developing better prognostic institution. Biomarkers for Circulating microRNAs (miRNAs) have been documented which directed the way to clinical applications. Here, to devise finest investigation method. For devising and profiling of microRNA, 4046 serum samples, including 428 patients with ovarian tumors, researchers used lab-on-chips. A diagnostic model, depends on expression of ten microRNA, is being manufactured in discovery set. This model is precise (sensitivity, 0.99; specificity, 1.00), and in ovarian cancer symptomatic accuracy was retained. moreover, scientists made more models, using 9-10 serum miRNAs used in all, targeted at diagnose ovarian cancers from the other types of ovarian tumors solid tumors or benign. Our result showed that profile the serum miRNA represent ensures investigative biomarker for cancer in ovaries [2].

\section{Exposing A Virus Hiding in The Animal Facility}

In laboratory mice sample it was recognized that kidney diseases were characterized by sudden nephropathy. It is caused by viruses called as mouse kidney parvovirus. The sequence of RNA sample was extracted from immunodeficient mice. The mice were facing from kidney fibrosis and renal failure. It was observed that some sequences were related to the sequence of parvovirus. The virus known as mouse kidney immuno deficient mice, could not be separated in culture. These viruses were transmitted to virus which are immuno deficient, through infected material. The experiment concluded the seriousness of disease, virus was replicated in the tissues of kidney. Also, shortly discussed that how the virus can be exposed which never usually detected by diagnostic test. Symptomatic test tells us about the detection. Result may vary in the infected mice by contamination. These results were compared with uninfected animals. So, viruses can affect the laboratory environment and experiment system. The immune responses will alter as compared to unsterile mice. These viruses are commonly present in colonies of mouse and the tools not detect them. The detection of these viruses may lead to understand the pathology of tubule interstitial fibrosis [3]

\section{Role of Bacterial Siderophores In Promoting Animal Host Iron Accession and Growth}

Siderophore is a Greek word means Iron Carrier. These are the small chelating compounds having high affinity for iron in ferric form. These compounds are secreted by bacteria and fungi, involve in transporting iron across the cell membrane. Iron has a main role in the virulence of microbes and in the defense provided by the immune system of the animal host. As both the host and pathogen require this crucial element, to explain its significance Qi and Han in
2018 described that the siderophores are involved in gaining iron for bacteria or pathogen and then the iron is utilized by the host or animal for growth. The common requirement for iron has also been explained by using a bacterivore named as $C$. elegans which need bacteria for growth and development. The $C$. elegans used in experiment grown on agar plates on which live bacteria were propagated. Such experiments were also used for determination of the required amount of vitamin B2 for growth (Qi et al. and Watson et al.). By using these experimental approaches researchers have examined the requirement of the micronutrients present in different bacteria which are phagocytosed by C. elegans. They have found five different genes for the entero bactin siderophore by using different mutants of E. coli bacteria while checking the effect of these mutants on the growth and development of $C$. Elegans. From the experiments researchers also came to know about the significance of entero bactinsiderophore. They found that the absence of entero bactin in the mutant $E$. coli has a significant impact on the growth of $C$. elegans, but the mutation in entero bactin receptor named as FepA has no effect. Furthermore, the entero bactin siderophore with heat-killed bacteria has no role because in heat killed bacteria there is a lack of micronutrients necessary for growth of $C$. elegans [4].

\section{Evolutionary Pressure against MHC Class II Binding Cancer Mutations}

MHC-I-restricted cytotoxic CD8+ T cell immune responses is shown in cancer and mutated peptides. MHC-I relation to neoantigen selection and tumor evolution is very broad range. MHC-II CD4+ T cells also make anti-tumor responses, but their mechanism is not extensively studied. the MHC-II genotype limit the mutational during tumor genesis. MHC-II act complementary to MHC-I. MHCII is selected during mutations while MHC-I are scantily selected. It shows that CD4+ T cells play important role in anti-tumor immunity. These effect of MHC-I and MHC-II were observed according to age. Figure 1 generally elaborate the process [5].

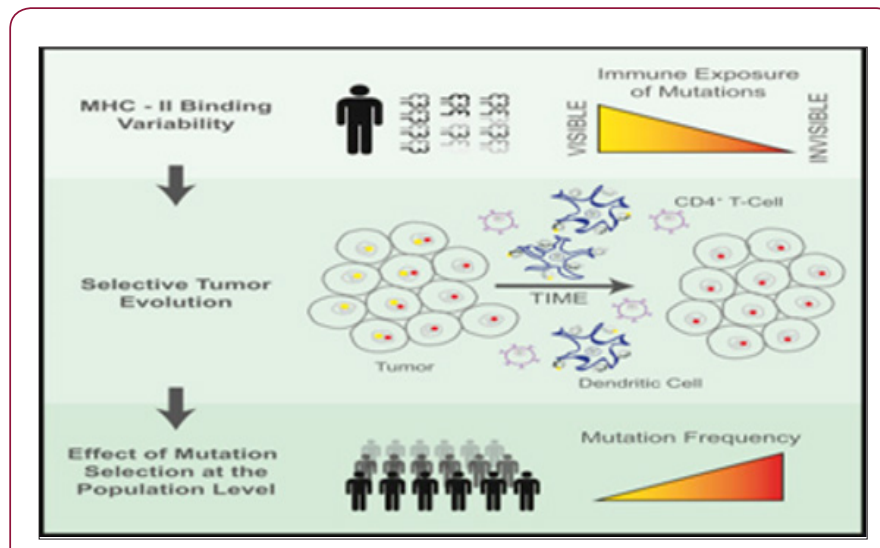

Figure 1: Selective Tumor Evolution and MHC-1 Complex.

\section{References}

1. Ni Kaiyuan, Guangxu Lan, Samuel S Veroneau, Xiaopin Duan, Yang Song, et al. (2018) Nanoscale metal-organic frameworks for mitochondriatargeted radiotherapy-radiodynamic therapy. Nature communications 9(1): 4321. 
2. Yokoi A, Juntaro Matsuzaki, Yusuke Yamamoto, Yutaka Yoneoka, Kenta Takahashi, et al. (2018) Integrated extracellular microRNA profiling for ovarian cancer screening. Nature communications 9(1): 4319.

3. Parrish CR (2018) Exposing a Virus Hiding in the Animal Facility. Cell 175(2): 310-311.

\section{ISSN: 2574-1241}

DOI: $10.26717 /$ BJSTR.2018.11.002053

Muhammad Irfan. Biomed J Sci \& Tech Res

(C) (i) This work is licensed under Creative

Submission Link: https://biomedres.us/submit-manuscript.php
4. Kim DH (2018) Bacterial Siderophores Promote Animal Host Iron Acquisition and Growth. Cell 175(2): 311-312.

5. Marty R, Wesley Kurt Thompson, Rany M Salem, Maurizio Zanetti, Hannah Carter (2018) Evolutionary Pressure against MHC Class II Binding Cancer Mutations. Cell 175(2): 416-428.

$\begin{array}{ll}\text { BIOMEDICAL } & \text { Assets of Publishing with us } \\ \text { RESEARCHES } & \text { - Global archiving of articles } \\ & \text { - Immediate, unrestricted online access } \\ \end{array}$

